\title{
Percutaneous closure of paravalvular prosthetic mitral leak requiring acute hemodialysis
}

\author{
Fabiola Sozzi ${ }^{1}$, Franck Levy ${ }^{2}$, Francois Bourlon ${ }^{2}$, Nicolas Hugues ${ }^{2}$, Laura Iacuzio ${ }^{2}$, Philippe \\ Rossi $^{2}$, Armand Eker ${ }^{2}$, Marco Schiavone ${ }^{1}$, and Filippo Civaia ${ }^{2}$ \\ ${ }^{1}$ Fondazione IRCCS Ca' Granda Ospedale Maggiore Policlinico \\ ${ }^{2}$ Centre Cardio-Thoracique de Monaco
}

November 19, 2020

\begin{abstract}
A case of a severe paravalvular mechanical mitral prosthesis leak in a high-risk patient that underwent previously 4 mitral valve surgeries is described. An Amulet-Amplatzer device was correctly implanted on the left atrial appendage, with the temptative to close also the contiguous severe leak. A residual moderate paravalvular leak caused acute hemolysis with severe renal failure requiring hemodialysis. The transesophageal echocardiogram is crucial in localizing and defining the extent of the paravalvular leak. It is not clear if the hemolysis was precipitated by the evolution of the disease or by the residual regurgitation after Amulet device deployment. Certainly, given the intricacy of the procedure the results might be limited also for highly expert operators. To our knowledge, this is the first case reporting the use of the Amulet-Amplatzer device for paravalvular leak closure.
\end{abstract}

\section{Percutaneous Closure of Paravalvular Prosthetic Mitral Leak}

\section{Requiring Acute Haemodialysis}

Fabiola Sozzi MD*, PhD; Frank Levy MD; Francois Bourlon MD; Nicolas Hugues MD,

Laura Iacuzio MD; Philipe Rossi MD, Armand Eker MD; Marco Schiavone MD*, Filippo Civaia MD

Cardiothoracic Centre CCM, Monaco, MC

*IRCCS Fondazione Ospedale Policlinico Ca' Granda, Milan, IT

\section{Address for correspondence:}

Fabiola Sozzi

Cardiothoracic Centre

11 bis, Avenue d'Ostende

98000 Monaco, MC

+39 (329) 5662258

fabiola_sozzi@yahoo.it

\section{ABSTRACT}

A case of a severe paravalvular mechanical mitral prosthesis leak (PVL) in a high-risk patient that underwent four mitral valve surgical replacements is described. A percutaneous PVL closure procedure was performed. An Amulet-Amplatzer device was implanted on the left atrial appendage correctly, and the temptative to 
close also the contiguous severe PVL was carried out. New-onset hemolysis post-PVL closure due to residual device leakage occurred. A severe renal failure requiring hemodialysis was developed.

An early haemolytic anemia can appear after a technically successful percutaneous PVL closure. It is due to a residual leakage around the device. When the hemolysis is severe, requires transfusions, and necessitates closure of the residual leak, device exchange, or device removal. This case required device removal and suture of the leak.

The results of PVL percutaneous closure might be limited also for highly expert operators, given the potential intricacy of the procedure. The transesophageal echocardiogram is crucial in defining the extent of the PVL and mandatory for guiding the leak closure procedure.

To our knowledge, this is the first case reporting the use of the Amulet-Amplatzer device for a percutaneous PVL closure.

Keywords: Paravalvular leak, Prosthesis mechanical mitral valve, Percutaneous closure, Amulet-Amplatzer device.

A 69-year-old woman underwent four mitral valve surgical replacements, the most recent one 11-years prior with a $27 \mathrm{~mm}$ mechanical bileaflet Sorin prosthesis. She presented with congestive heart failure symptoms and atrial fibrillation. A significant paravalvular prosthetic mitral leak (PVL) was shown at transthoracic echocardiogram (TTE) [Figure 1/Video 1]. Transesophageal echocardiogram (TEE) regarded the PVL as major, flowing into the large left atrial appendage (LAA) [Figure 2/Video 2]. A partial prosthesis disconnection (sized $10 \times 4 \mathrm{~mm}$ ) with stitches dehiscence and a postero-lateral leak tunnel, immediately next to a large funnel-shape LAA was shown at the 3D-reconstruction (Figure 3/Video 3). The patient denied fever or other constitutional symptoms. Laboratory studies showed normal haemoglobin, haematocrit, renal function, and negative blood cultures. Because of multiple prior sternotomies, it was elected to proceed with a percutaneous PVL closure. Considering the contiguity of the PVL to the LAA an Amplatzer-Amulet LAA device of $28 \mathrm{~mm}$ was chosen. The LAA was large (landing zone of $24 \mathrm{~mm}$ ) and it was hypothesized the possibility to cover also the PVL with the disk (diameter of $34 \mathrm{~mm}$ ). The procedure was performed via anterograde trans-septal approach under TEE guidance with general anesthesia (Figure 4/Video 4). The prosthesis was properly released on the LAA (Figures 5-6/Videos 5-6) and deemed the best as possible for the closure of the contiguous PVL. Unpredictably, the adhesion of the lobe occluder to the PVL wall was incomplete. Several attempts to close the residual PVL failed. Thus, a PVL with moderate regurgitation remained (Figures 7-8/Videos 7-8) and a hemodynamic worsening with acute hemolysis occurred. The severe hemolytic anemia was complicated by acute renal failure requiring hemodialysis and multiple blood transfusions. An emergency cardiac surgery necessitated, with removal of the Amplatzer-Amulet device and suture of the PVL. A subsequent clinical resolution was obtained. The first-year follow-up was negative for cardiac events.

\section{DISCUSSION}

This case describes a major postero-lateral PVL developing heart failure class IV, percutaneously closed with an Amplatzer-Amulet device. A severe haemolysis with acute renal failure requiring hemodialysis occurred early after the percutaneous procedure. To our knowledge, this is the first case reporting the use of Amplatzer-Amulet device for PVL closure.

The Amulet device was properly released and deemed the best as possible for the closure of the contiguous PVL, but the adhesion of the lobe occluder to the PVL wall was incomplete. The postprocedure TEE showed a moderate residual PVL that affected the immediate significant hemodynamic worsening with hemolysis for increased red blood cell shear stress, with mechanical trauma and fragmentation. The consequent acute kidney injury required hemodialysis and an urgent surgical revision with device removal was needed. It is not completely clear if the acute hemolysis was precipitated by the use of this particular device or the evolution of the turbulent flow through the moderate PVL. Certainly, before the attempt of PVL closure neither increased red blood cell fragility nor pre-existing anemia with increased turbulence were documented. 
PVL is a challenging complication of mitral valve replacement, associated with significant morbidity and mortality (1). As known, paravalvular defects usually result from poor tissue quality related to friability from infection or calcification around the prosthetic valve. Although most PVLs are subclinical, about $3 \%$ of patients develop severe heart failure, hemolysis, or a combination of both, necessitating intervention. Hwang et al, analysed a population of 1202 patients who underwent mitral valve replacement and followed them over a mean period of 134 months. Only a subgroup of 55 patients developed late major PVL without obvious infection, with 10- and 20-year major PVL-free rates of $96.2 \%$ and $86.9 \%$, respectively (2). The last ACC/AHA guidelines for the management of valvular heart disease recommended percutaneous repair of PVL (Class IIa) for high-risk surgical patients with either intractable hemolysis or NYHA Class IIIIV heart failure who have anatomic features suitable for catheter-based therapy and when performed in centres with high expertise (3). Successful PVL closure is defined as reduction of regurgitation to the lowest possible grades (grade [?]1) which translates into superior clinical outcomes (4). Alkhouli et al, retrospectively analysed 231 patients who underwent percutaneous closure of mitral PVL with Amplatzer (73\% with Vascular Plug II, the remaining devices included Duct Occluder, Septal Occluder, and Muscular VSD Occluder) in a 10-years period (2006-2017) and found that successful percutaneous reduction of the PVL to mild or less was associated with significant midterm survival benefit (5). The data from the UK PVL showed the percutaneous closure of 308 PVL in 259 patients, in 10-years period (2004-2015) and found significant reduction of death and major adverse cardiovascular events. In general, the most commonly used occluder devices include the Amplazer vascular plug family, comprising the type II, III and IV, the Amplatzer duct occluder, the muscular ventricular septal defect occluder, and the paravalvular leak device (6).

In our patient a careful pre-procedural planning and evaluation of diagnostic imaging as well as knowledge of prosthetic valves was acquired. Skills in complex catheter techniques was also present. To the best of our knowledge, this is the first case that describes the attempt to close the PVL using the Amulet device. Indeed, the crucial role of TEE in localizing and defining the PVL extent is known, given the high shadowing created by the sewing ring, that obscures and limit TTE. Additionally, TEE was mandatory for guiding the leak closure procedure.

\section{CONCLUSION}

In high surgical risk patients, the percutaneous closure of PVL is a promising alternative. The appropriate approach is not always standardized. Unpredictable factors can make the procedure failing.

\section{REFERENCES}

1. Eleid MF, Cabalka AK, Malouf JF, et al. Techniques and outcomes for the treatment of paravalvular leak. Circ Cardiovasc Interv.2015;8:e01945.

2. Hwang H-Y, Choi J-W, Kim H-K et al. Paravalvular leak after mitral valve replacement: 20-year follow-up. Ann Thorac Surg.2015;100:1347-52.

3. Nishimura RA, Otto CM, Bonow RO, et al. 2014 AHA/ACC Guideline for the management of patients with valvular heart disease: executive summary: a report of the American College of Cardiology/American Heart Association Task Force on Practice Guidelines. Circulation.2014;129:2440-92.

4. Okutucu S, Mach M, Oto A. Mitral paravalvular leak closure: transcatheter and surgical solutions. Cardiovasc Revasc Med. 2019;pii:S1553-8389(19)30372-0.

5. Alkhouli M, Zack CJ, Sarraf M, et al. Successful percutaneous mitral paravalvular leak closure is associated with improved midterm survival. Circulation: Cardiovascular Interventions . 2017;10:e00573.

6. Calvert PA, Northridge DB, Malik IS, et al. Percutaneous device closure of paravalvular leak combined experience from the United Kingdom and Ireland. Circulation. 2016;134:934-944.

\section{Figures' Legend}

Figure 1, Video 1: Transthoracic echocardiogram (TTE): A turbulent eccentric jet originating from outside the prosthetic sewing ring compatible with a paravalvular leak (PVL) is shown. A gap is visualized between the annulus and the sewing ring. A limitation for prosthetic valves evaluation at the TTE is the shadowing determined by the sewing ring, which obscures color-flow Doppler images. 
Figure 2, Video 2: Color-Doppler transesophageal echocardiogram (TEE) demonstrates a major PVL flowing into the left atrium (LA) towards the large left atrial appendage (LAA).

Figure 3: Color 3D-TEE shows a postero-lateral leak tunnel due to stitches dehiscence and the partial mitral valve (MV) prosthesis disconnection.

Video 3: 3D-TEE images of bileaflet mechanical mitral valve. Surgical view of the MV (viewed from the left atrial side).

Figure 4, Video 4: TEE guidance during fluoroscopic images of the Amulet device deployed across the PVL.

Figure 5, Video 5: 2D-TEE showing the proper deployment of the prosthesis over the LAA. LV = left ventricle.

Figure 6, Video 6: 3D-TEE showing the proper deployment of the prosthesis over the LAA.

Figure 7, Video 7: Color 2D-TEE shows incomplete adhesion of the lobe occluder to the PVL wall. Therefore, a residual moderate PVL is demonstrated.

Figure 8, Video 8: Color 3D-TEE: The postero-lateral PVL with moderate regurgitation is visualized. It caused hemodynamic worsening with acute hemolysis.

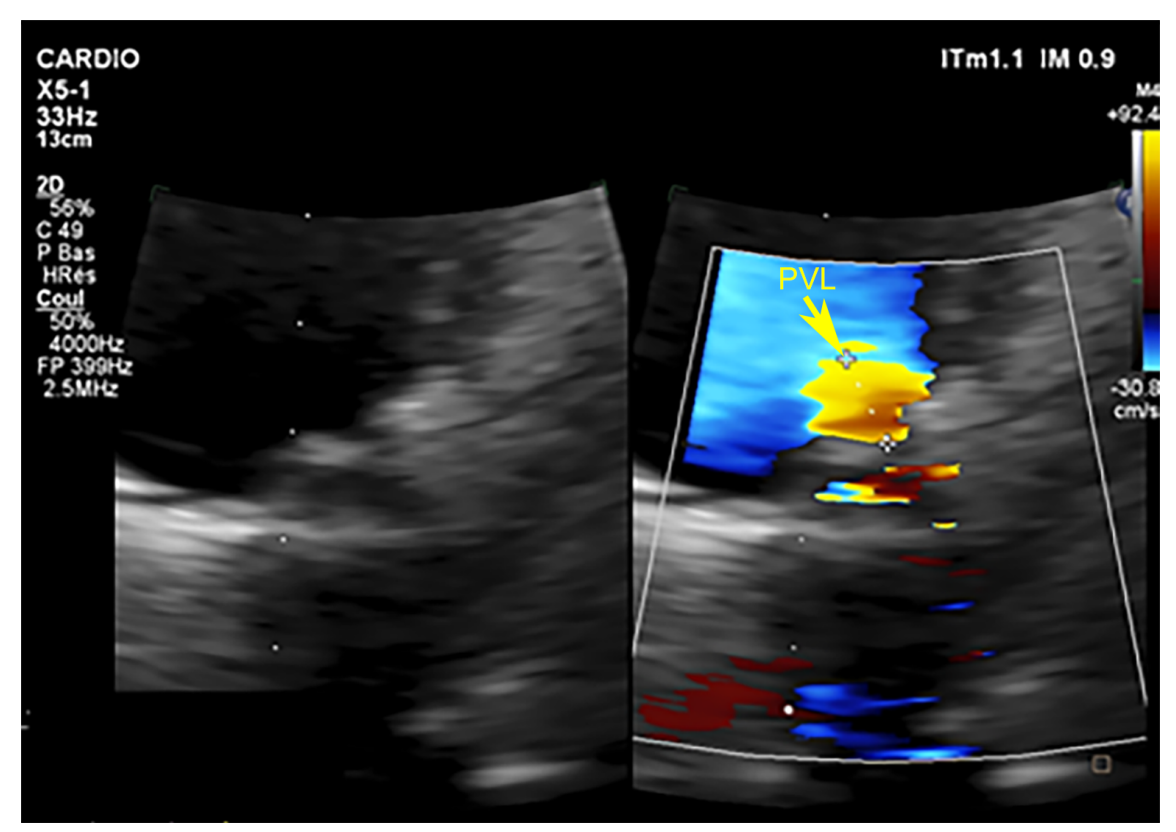



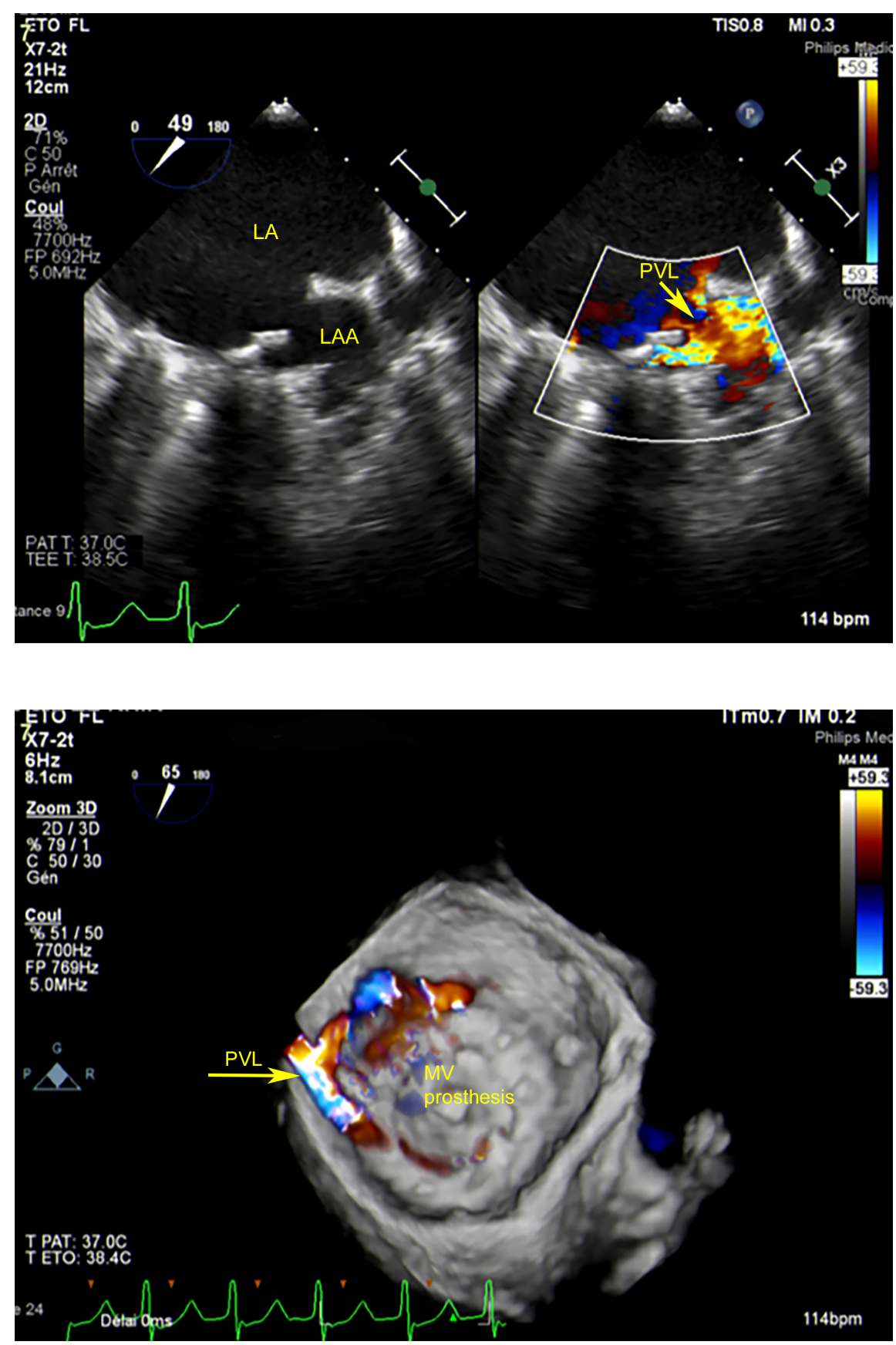

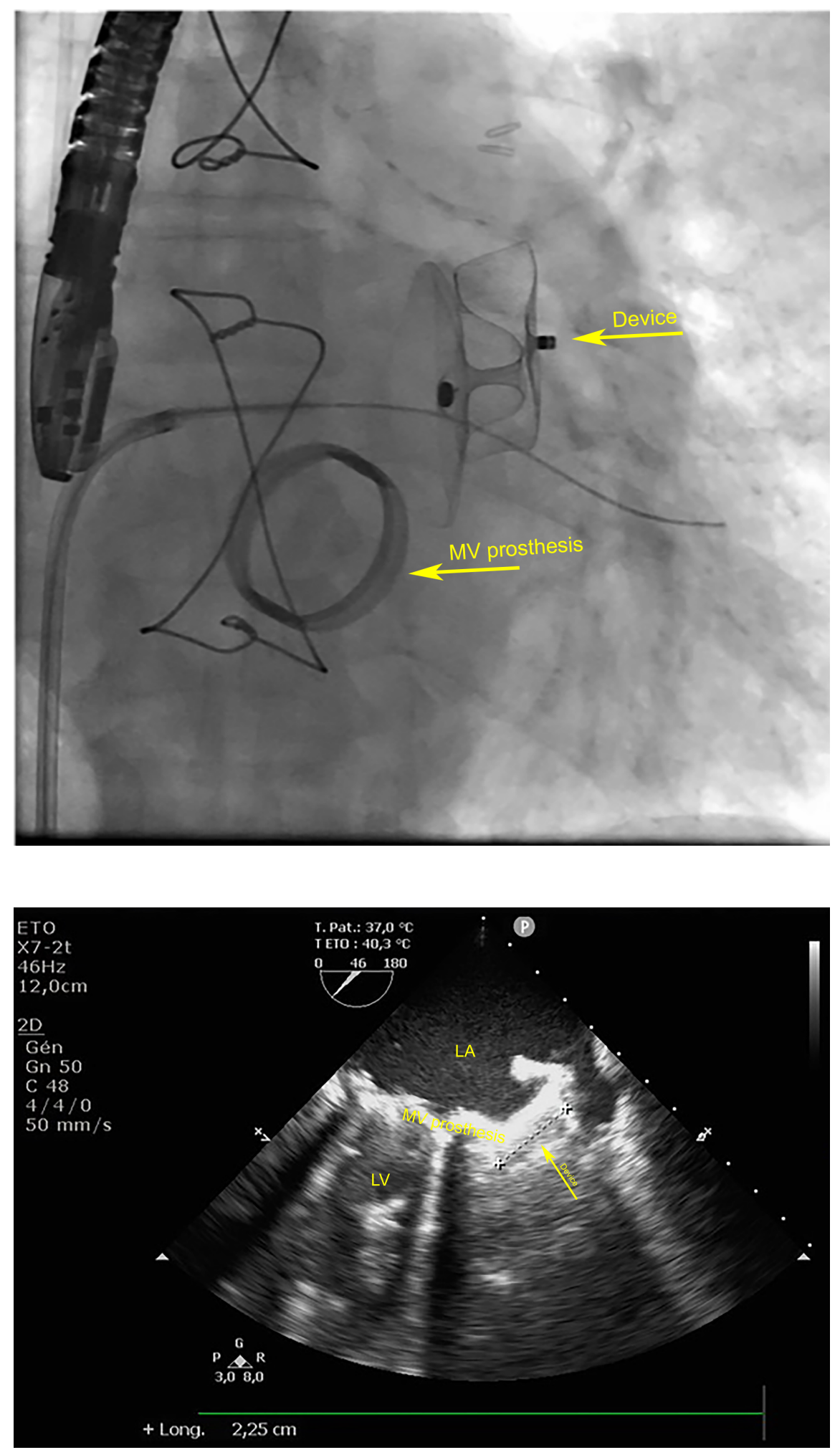

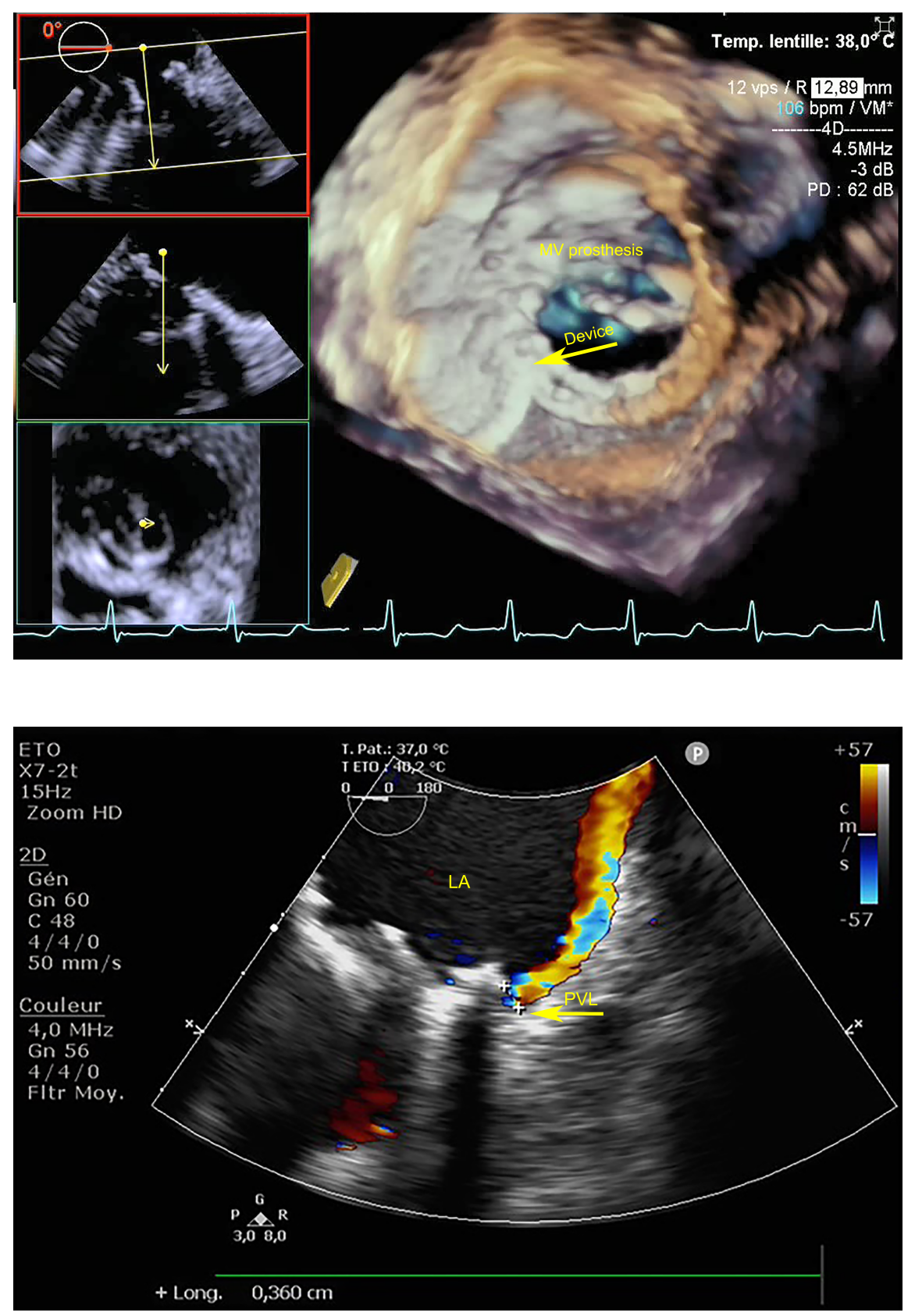


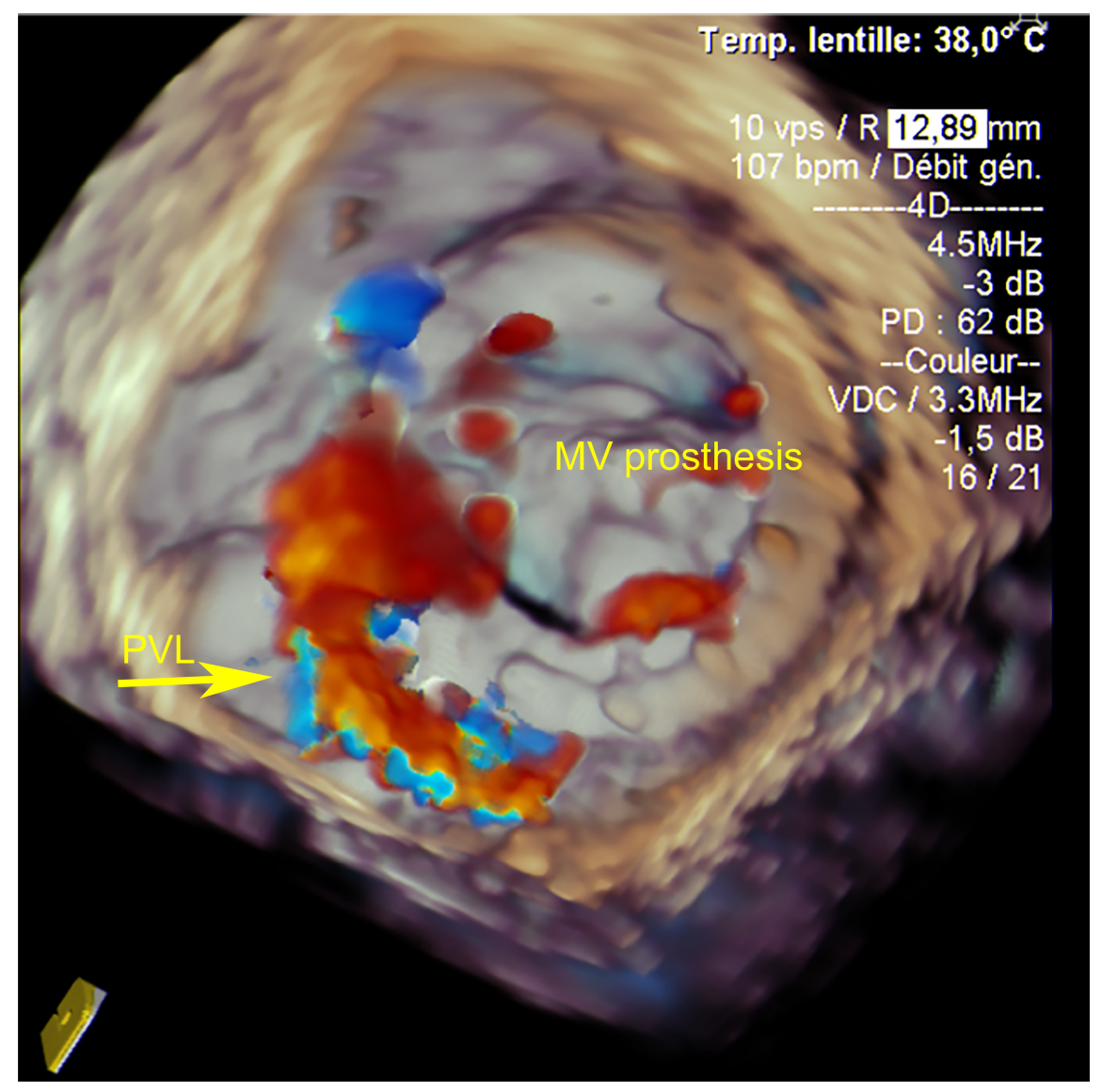

